\title{
Intelligent Distance Measurement of Robot Obstacle Avoidance in Cloud Computing Environment
}

\author{
Zhili Zhang ${ }^{*}$, Chunping Liu, and Xiaoming Ma \\ Intelligent Manufacturing College, Tianjin Sino-German University of Applied Science, Tianjin, 300350, China
}

\begin{abstract}
The application of robots plays an important role in the development of intelligent production and life. At present, most robots avoid the obstacle in the process of robot operation through a single ultrasonic ranging, and it cannot guarantee the accuracy of the obstacle avoidance of robots. In this paper, an intelligent distance measurement method of robot obstacle avoidance in a cloud computing environment is designed and studied. Based on the DSP and ultrasonic global positioning system, a multi-channel ultrasonic transmitter/receiver module is adopted to design an autonomous obstacle avoidance control system based on ultrasonic waves and a new fuzzy reasoning method is proposed to realize the function of intelligent distance measurement of robot obstacle avoidance in the cloud computing environment. The simulation and field test for the intelligent distance measurement system of the obstacle avoidance is carried out by Visual C ++ visual programming software. The reliability and feasibility of the system are verified, which provides a wider space for the research and development of the robot.
\end{abstract}

Keywords: cloud computing; robot; obstacle avoidance

(Submitted on November 6, 2018; Revised on December 5, 2018; Accepted on January 3, 2019)

(C) 2019 Totem Publisher, Inc. All rights reserved.

\section{Introduction}

Robots and other intelligent industries are as the greatest areas of the 20th century, which are used widely. In the 20thcentury development process, the core of the development of the robot is that the obstacle avoidance distance measurement technology continues to improve. With the rapid development of computer technology, advanced sensor technology and a variety of automated intelligent control in 21 st century, automation, intelligentialization is the development theme of robots and other intelligent industries [1]. The development trend of the robot industry has the following important significance. First, with the development of robot intelligentialization, the robot not only has a simple life tool, but also plays a more important and more intelligent role in people's lives, which is in line with the theme of modern smart homes. Second, the development of intelligent robots can greatly reduce the consumption time of modern people in household labor and other aspects. The intelligent applications can ease the tension of modern life to a certain extent [2-3]. Therefore, the development of intelligent distance measurement of obstacle avoidance will be a milestone in the history of robot development [4-5].

Jong-Wooket al. proposed an automatic trajectory generation method of robot [6]. On the basis of the robot model, the position and attitude of the corresponding robot are calculated by using the kinematic method and the optimal solution is selected to realize the automatic selection path of the robot in the obstacle and non-obstacle environment and the path optimization of the mobile robot. The algorithm improves the attitude performance, is stable and reliable, and has certain engineering significance. However, the real-time requirement is not strong.It cannot be used in the intelligent distance measurement of obstacle avoidance. An application method for the intelligent distance measurement of obstacle avoidance by using reinforcement learning based on neural network is proposed by Benzaoui et al. [7]. Based on the neural network reinforced Q-learning algorithm, the intelligent control structure is integrated to apply to the obstacle avoidance of mobile robots. The algorithm has a certain guiding significance since the robot is the object of study, but the model is not fineenough and cannot fully reflect the characteristics of the robot obstacle avoidance distance measurement. Luo et al. used

\footnotetext{
* Corresponding author.

E-mail address: asdf147852a1@163.com
} 
a formation control method of intelligent distance measurement of obstacle avoidance for multi-mobile robots [8]. This method introduced the navigation function and used a kind of formation control algorithm for obstacle avoidance distance measurement to ensure that the formation movement does not collide with the obstacles. The algorithm has a good guiding significance for the formation, focusing on the overall effect. However, for a single individual, it did not have optimized performance. Ismail et al. proposed an obstacle avoidance distance measurement method of mobile robots based on fuzzy logic control [9]. This method used the fuzzy control algorithm which had the robustness and was based on the combination of perception and action in biology to achieve effective avoidance distance. However, the method had a certain degree of uncertainty, so it cannot be applied to high safety requirements systems of the obstacle avoidance distance measurement.

Regarding the issue above, we propose an intelligent distance measurement method of robot obstacle avoidance in a cloud computing environment. DSP and ultrasonic global positioning system are used as the basis and a multi-channel ultrasonic transmitter/receiver module is adopted to design an autonomous obstacle avoidance control system based on ultrasonic waves and a new fuzzy reasoning method is proposed to realize the function of intelligent distance measurement of robot obstacle avoidance in the cloud computing environment. Finally, the simulation and field test for the intelligent distance measurement system of the obstacle avoidance are carried out by Visual $\mathrm{C}++$ visual programming software. The reliability and feasibility of the system are verified, which provides a wider space for the research and development of the robot.

\section{Principle of Distance Measurement Algorithm for Robot Obstacle Avoidance}

In order to solve the problem of robot obstacle avoidance during operation, an intelligent distance measurement method of robot obstacle avoidance is proposed, which combines the information of the multi-sensor to fit the distance of the obstacle. The method of infrared transmission is used to determine the obstacle. Then, the information of the sensor is coded in one dimension, and the optimal obstacle avoidance path is taken by particle swarm optimization [10-11].

After the robot's start button is pressed, the sensor periodically collects the surrounding data. The data collected by the infrared sensor is whether the surrounding obstacles exist, and the data collected by the ultrasonic sensor includes the type of surrounding obstacles [12]. Data transmission of sensors use the bus transmission mode and the data through the bus technology can be a stable transmission. The transmitted information is stored in the storage device through the data processing. The drive circuit is commanded to avoid obstacles and to prevent the robot stuck phenomenon [13-16]. The design of the system is divided into three parts: driving mechanical design, data acquisition design, and obstacle avoidance distance measurement design.

\section{Part 1: Driving mechanical design}

The robot is a smaller size mechanical body, driven by the motor to run. The design of the wheel is a caster to ensure that the robot can be rotated [17], greatly improving the flexibility of the robot. Because robots are walking in environments where the environmental structure is highly random [18], the bottom distance of the different obstacles is not the same, so the size design should be as small as possible and easy to carry. The mechanical design of the wheel body includes the motor driven and the front and rear caster. The design of the caster ensures that the robot can be rotated in situ to improve the flexibility of walking. The mechanical body of the robot is made of aluminum material [19]. The motor is driven by a typical H-bridge switch circuit. This bridge technology is driven by a $5 \mathrm{~V} \mathrm{DC}$ motor to ensure the negative and the positive rotation of the motor.

\section{Part 2: Information collection section}

In this paper, the sensor installation and information collection are taken by the classic method of robot sensor installation, and the infrared sensor and ultrasonic sensor are installed around the robot. The ultrasonic sensor for the obstacle's distance detection is the use of an ultrasonic sensor (Version 600) made by the INC Co. Ltd., Polish. The infrared sensor and the receiving tube are used to realize the obstacle detection of the infrared sensor. Around the Robot, infrared sensors and ultrasonic sensors are equipped. The left and the right sensor can ensure that the robot will be accurate judgments when walking along in the wall and the side of obstacles. The ultrasonic sensor is a non-contact sensor. Because of its accuracy of distance detection, in the field of intelligent detection, this sensor is applied extensively. Its working principle is to rely on calculating the time of the forward and backward reflection of the ultrasonic waveform. From the ultrasonic wave generation to the echo perception by the receiver, according to the propagation time of waveform in the air, the distance can be measured as Equation (1), where $\mathrm{c}$ and $\mathrm{t}$ refer to the time and speed of the ultrasonic wave propagation respectively. The propagation velocity of the ultrasonic wave at constant temperature can be simply recorded as $340 \mathrm{~m} / \mathrm{s}$, and the distance between the sensor and the reflection point is shown as Equation (2). 


$$
\begin{gathered}
d=c t / 2 \\
d=\sqrt{s^{2}-\left(\frac{h}{2}\right)^{2}}
\end{gathered}
$$

In an intelligent robot, a lot of sensors are installed. Because of the emergence of blind spots, it causes a large number of signal crosstalk when these sensors collect the surrounding information. Signal crosstalk brings noise interference, and noise interference can cause a large degree of test error in the process of data fusion. A large part of these errors are unacceptable. The hardware design techniques used in this article will reduce the measurement error due to the crosstalk of the signal. In the multi-sensor acquisition system, the distance measurement method is mainly based on ultrasonic sensors and supplemented by infrared sensors. Because ultrasonic sensors cannot measure the closed distance, infrared sensors are used.Using the strobe circuit 74HC4051 and LM567 chip, the infrared drive circuit described above is built. The transmitting tube is in series andthe transmitting power is debugged by an appropriate adjustment of the resistance.

\section{Part 3: Design of distance measurement module of obstacle avoidance}

The distance measurement module of obstacle avoidance is an important manifestation of the intelligentization of the robot, which is responsible for driving the robot work and the mechanical body. As the robot's processor information is large, it should have the fast processing speed, and the control program of the processor should have a distinction of a variety of extended function interfaces and interrupt levels [20]. In this paper, the TMS320LF2407A chip is used for core control. The chip not only has a variety of serial interfaces but also has 32 storages, providing up to 40 expansion interfaces for system expansion and upgrades.

In this paper, the classical group intelligent search technology is used in the process of obstacle avoidance distance measurement and the intelligent inference is discussed. The particle swarm optimization algorithm is a modern optimization algorithm which imitates bird foraging. The basic idea is to encode the position search space of the particle as the exploration of the optimal solution. Assuming that the search space of the particle swarm is in the $D$ dimension, mapping in the path avoidance of the robot is the optimal path selection of obstacle avoidance. Assuming that the search space of the particle swarm is $D$-dimensional, the mapping in the robot path avoidance is the optimal path selection for obstacle avoidance. Assuming that the spatial position of the $i^{\text {th }}$ particle, that is, the driving path of the next robot, can be expressed as $X_{i}=\left(X_{i 1}, X_{i 2}, \cdots, X_{i d}\right)$.

$$
\begin{gathered}
V_{i d}^{k+1}=\omega V_{i d}^{k}+c_{1} r_{1}\left(p b_{i d}^{k}-x_{i d}^{k}\right)+c_{2} r_{2}\left(g b_{d}^{k}-x_{i d}^{k}\right) \\
X_{i d}^{k+1}=X_{i d}^{k}+V_{i d}^{k+1}
\end{gathered}
$$

In the optimization of the obstacle distance measurement by particle swarm optimization, the grid method can be used to model the environment. This method is used to make special markings for the obstacles after grid processing of the environment. The grid method in the modelling of a small grid will be standardized into a larger lattice modelling. The small grid in the grid modelling which is dissatisfied with a grid that will be standardized into a larger grid for modelling is shown in Figure 1.
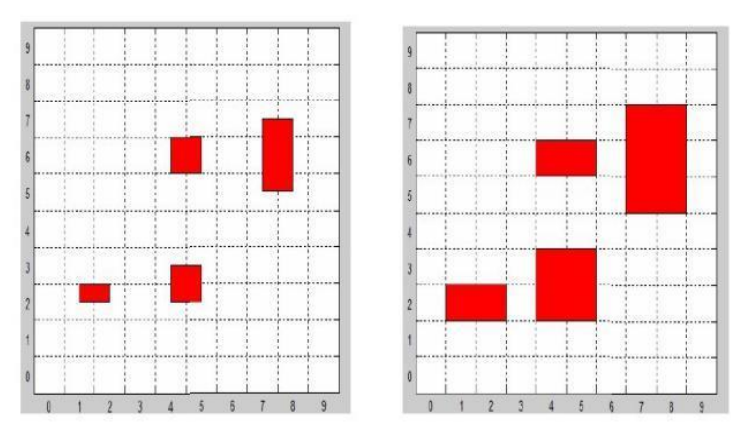

Figure 1. Schematic diagram of grid modelling

After perceiving the data by the obstacle sensor, a two-dimensional coordinate is formed. In this paper, in order to 
reduce the information processing delay of the two-dimensional coding, the two-dimensional coding is simplified to remove abscissa information like Figure 2.

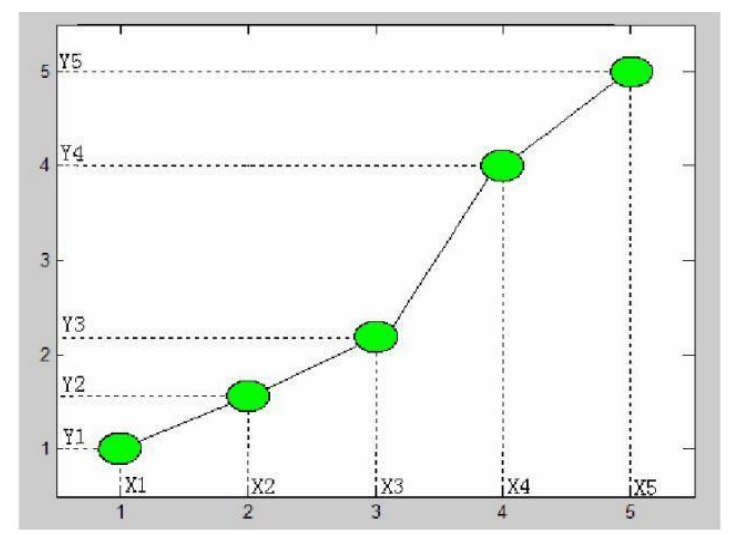

Figure 2. Schematic diagram of robot path coding

In the method of particle swarm optimization, the fitness function of the path should meet the condition of the path that is the shortest. The angle between the obstacle and the robot can be measured by the sensorand it is not necessary to join into the optimal selection of the path. The fitness function can be expressed as the following formulas, where $(X, Y)$ is the individual gene, $(x, y)$ is the obstacle coordinate, and the two objective functions can be effectively combined by the weighting factor. In the surrounding path search, after moving around along in the wall with a circle, the robot enters the working mode so the robot can avoid the obstacles under the support of the obstacle avoidance distance measurement algorithm.

$$
\begin{gathered}
f_{1}=1+f_{1}\left(X_{i}, Y_{i}\right)=\left(x_{j}, y_{j}\right), \quad i, j=1,2, \cdots, m \\
f_{2}=\sqrt{\left(X_{i}-X_{i-1}\right)^{2}+\left(Y_{i}-Y_{i-1}\right)^{2}}, \quad i=2,3, \cdots, n \\
F=w_{1} f_{1}+w_{2} f_{2}
\end{gathered}
$$

\section{Principle of Optimization Method for Intelligent Distance Measurement of Robot Obstacle Avoidance in Cloud Computing Environment}

\subsection{Principle Description for Distance Measurement of Robot Obstacle Avoidance}

The optimization method of obstacle avoidance distance measurement in the cloud computing environment is mainly based on the ultrasonic positioning. The ultrasonic positioning system is composed of multiple sensors which form a system that is widely used in the local positioning of the robot. The system is often composed of multiple receivers and a transmitter. The transmitter is fixed on the robot. According to the function of ultrasonic ranging, it can locate the object. The schematic diagram is shown in the following figure. $L_{1}$ and $L_{2}$ are placed in a receiver respectively, and the midpoint of the $L_{1}$ and $L_{2}$ connection is the origin $O$. A transmitter is placed on the robot $S$ and using the three-point method can measure the coordinate, where, $x_{s}$ and $y_{s}$ are the horizontal and vertical axis of the agricultural robot.

$$
\left\{\begin{array}{l}
y_{s}=\frac{D_{S L_{1}}^{2}-D_{S L_{2}}^{2}}{4 L} \\
x_{s}= \pm \sqrt{\frac{D_{S L_{1}}^{2}+D_{S L_{2}}^{2}-2 L^{2}-2 y_{s}^{2}}{2}}
\end{array}\right.
$$

The positive and negative values of $x_{s}$ are determined by the position of the point $O$ and the robot. The principle of the positioning system is to place the transmitter on the measured object and place the receiver (base point) on the nearby location. Then, send the signal by the robot needed to make positioning and receive the signal by the base point. The 
distance between each base point to the robot is calculated and through the wireless transmission module, the data can be sent to the DSP processor to calculate the robot coordinates like in Figure 3.

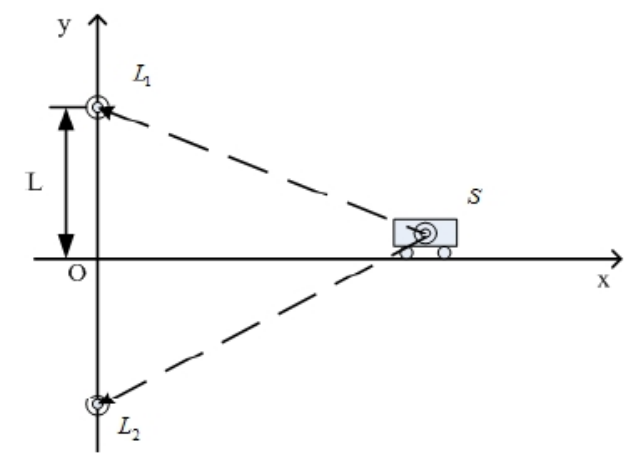

Figure 3. Schematic diagram of ultrasonic positioning system

In this paper, the ultrasonic positioning system is designed to place the transmitter on the robot and the receiver is placed on the nearby object as the base station. The transmitter transmits the signal and the fixed base station receives and measures the distance and sends it to the processor to find the robot coordinates. Using the three-ball positioning technique, the calculation model of the system can be obtained as shown in the following Figure 4.

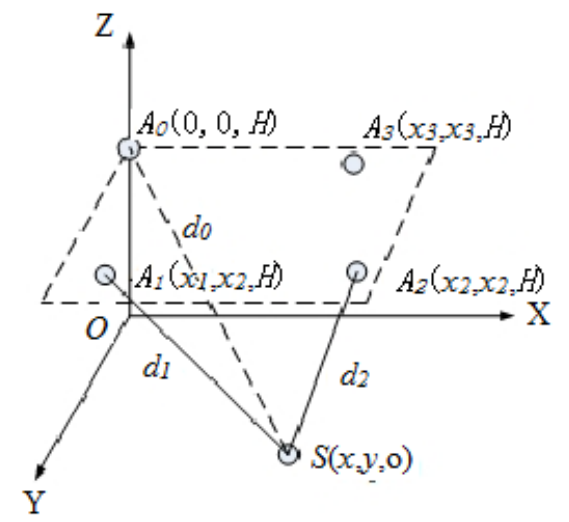

Figure 4. The calculation model of three-ball positioning principle

As shown above, three receivers as base stations are installed in the laboratory ceiling. Supposing that the location of the three base stations are $A_{0}(0,0, H), A_{1}\left(x_{i}, y_{1}, H\right), A_{2}\left(x_{2}, y_{2}, H\right)$, the coordinate of the robot on the horizontal ground is $S(x, y, 0)$. The following three-sided calculation formula can be used:

$$
\left\{\begin{array}{l}
\left(x-x_{0}\right)^{2}+\left(y-y_{0}\right)^{2}+H^{2}=d_{0}^{2} \\
\left(x-x_{1}\right)^{2}+\left(y-y_{1}\right)^{2}+H^{2}=d_{1}^{2} \\
\left(x-x_{2}\right)^{2}+\left(y-y_{2}\right)^{2}+H^{2}=d_{2}^{2}
\end{array}\right.
$$

In the above formula, $H$ is the height of the ceiling and $d_{0}, d_{1}$, and $d_{2}$ represent the distance between $A_{0}, A_{1}, A_{2}$, and the robot. Using the above formula, the robot coordinate $S(x, y, 0)$ can be solved.

\subsection{Design of Distance Measurement System for Robot Obstacle Avoidance}

In the development of robots, the automatic obstacle avoidance control is generally carried out in the case that the environmental map is predetermined. However, in a practical application, robots often run in an environment that is unknown or where the map is not accurate, which often causes too much difference between the previous map information and the sensor's actual perception. Therefore, it is very important for the robot to detect the front obstacle in real time and re-plan the path. With the increasing number of robotic applications, the ability of flexible obstacle avoidance is more and more important. It is an important condition of robots to complete the navigation tasks in complex environments. The 
distance measurement system for robot obstacle avoidance is mainly composed of detection systems, information processing, and the way of obstacle avoidance distance measurement.

Because robots need to move independently in an unknown environment, they need to have the capacity to detect and avoid obstacles by themselves. The ultrasonic transmitter and receiver module perceive the obstacle and its distance in a reflective way and can provide accurate information reference for the distance measurement of robot obstacle avoidance because of its small interference. This is due to environmental factors, accurate detection, and measurement of obstacle and distance at night. In the distance measurement system of robot obstacle avoidance, the use of a multi-channel ultrasonic sensor achieves an automatic obstacle avoidance distance measurement. Since the system designed in this paper includes two parts, positioning and obstacle avoidance distance measurement, the DSP processor processing task is arduous. In order to reduce the burden and improve the running speed of the system, this design adopts the fuzzy reasoning control technology and classifies and stores them according to the multi-channel ultrasonic detection information. On the basis of the fuzzy control, the robot makes real-time path planning.

In order to realize the intelligent distance measurement of obstacle avoidance, five sets of ultrasonic transmitting and receiving modules are installed in the front, left and right directions of the robot and so on. The module sends the ranging information to the DSP processor and then establishes the fuzzy rule base to determine the motion path and the moving direction of the robot according to the distance measurement result of robot obstacle avoidance.

\subsubsection{Hardware Design of Robot Positioning and Obstacle Avoidance Distance Measurement System}

The core processor used in this paper is the TMS320F2812 processor designed by the TI Co. Ltd. It possesses strong data processing capabilities, a fast running speed, and rich storage resources of SARAM and Flash chip. Based on the composition of the sensor's receiving module and the working characteristics of the DSP, a positioning and obstacle avoidance measurement system based on DSP and ultrasonic ranging is proposed. The hardware frame of the positioning and obstacle avoidance measurement system is shown in Figure 5.

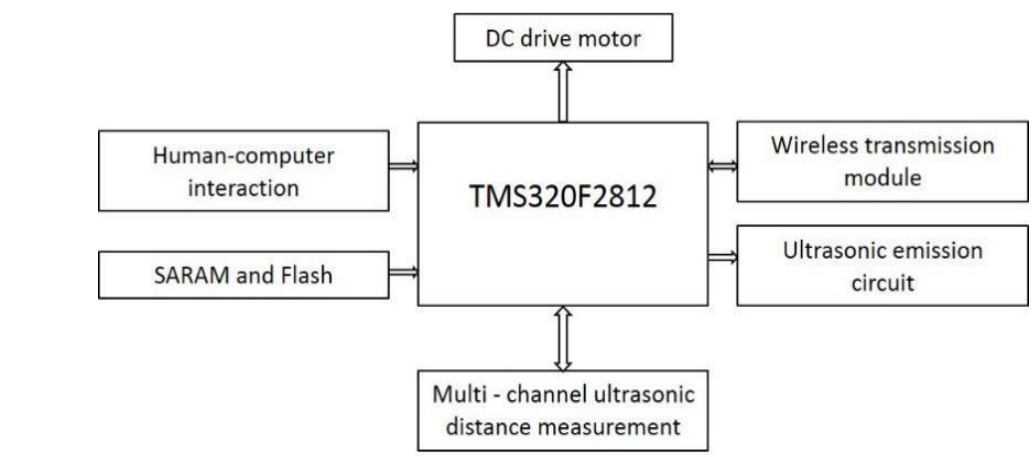

Figure 5. Hardware frame diagram of positioning and obstacle avoidance distance measurement system

As shown in Figure 5, the system is built on the robot test platform. The system uses the TMS320F2812 processor as the control core and integrates the wireless transmission module, the DC drive motor, the distance measuring sensor, and the ultrasonic transceiver module to realize the function of robot positioning and the obstacle avoidance distance measurement.

\subsubsection{Software Design of Positioning and Distance Measurement System for Obstacle Avoidance of Robot}

In the positioning system of the robot, signals are mainly transmitted by the transmitting module and then received by the receiving base station. After processing the data information by the base station, the data is transmitted by the wireless module to the processor, and then, according to the distance between each base point and the robot, the three-ball positioning principle is used to find the specific coordinate position of the robot.

After the system is powered on, the program starts to initialize and sets a series of parameters. Then, the main program calls the positioning subroutine. The subroutine calculates the coordinate value according to the collected data information and determines the specific position of the robot. Software design of the distance measurement system for robot obstacle avoidance mainly includes the main program, external interrupt, distance calculation, obstacle avoidance strategy and other subroutines. 


\section{Experimental Results and Analysis}

In order to test the performance of the algorithm in the distance measurement of robot obstacle avoidance, the simulation experiment is carried out. The simulation scene of the robot's behavior trace is simulated by the NetLogo simulation software. The robot used in this paper is the independent research and development of six degrees of the freedom $6 \mathrm{R}$ industrial robot which is equipped with a positioning sensor for sensing the distance information, environmental information and other experimental environments. The host computer is equipped with a Pentium (R) D CPU2.80GH, 2.79 GHz, and 2.00GB memory. In the experiment, the coordinate information of the walking path of the environment robot is set, including the indoor positioning sign and the probability center of the obstacle occurrence. The robot moves in a twodimension simulation plane with the size of $300 \times 300$ to make positioning and path deviation correct. In the simulation plane, the starting point of the robot's motion coordinate is set as [20, 20], [250, 230]. First, the robot's behavior space is made with mathematical modeling. Then, the robot's motion parameters are analyzed to get the number of behavior quantum $m=100$ in the distance measurement process of robot obstacle avoidance. The pheromone evolution factor $\alpha=1$, the expected heuristic value $\beta=7$, the pheromone volatility coefficient $\rho=0.3$, and the pheromone increase intensity of robot obstacle avoidance distance measurement $Q=1$. There are six rectangular obstacles objects and the size of the robot's behavior map is $300 \times 300$ pixels as shown in Figure 6 .

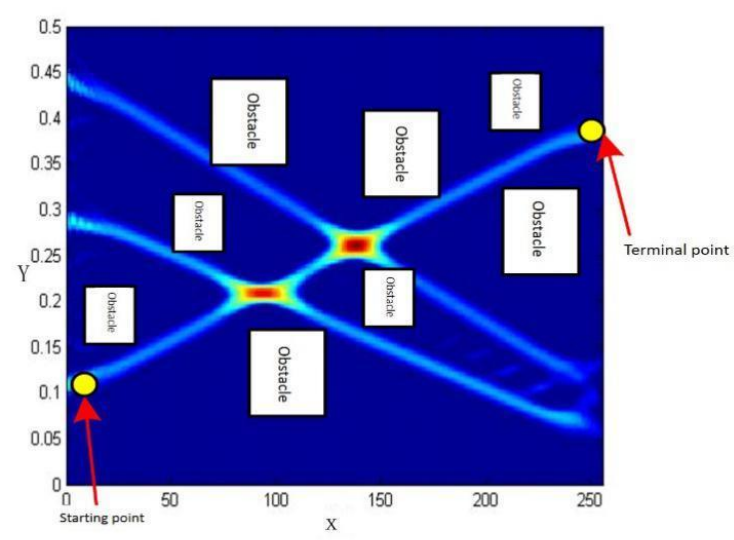

(a)

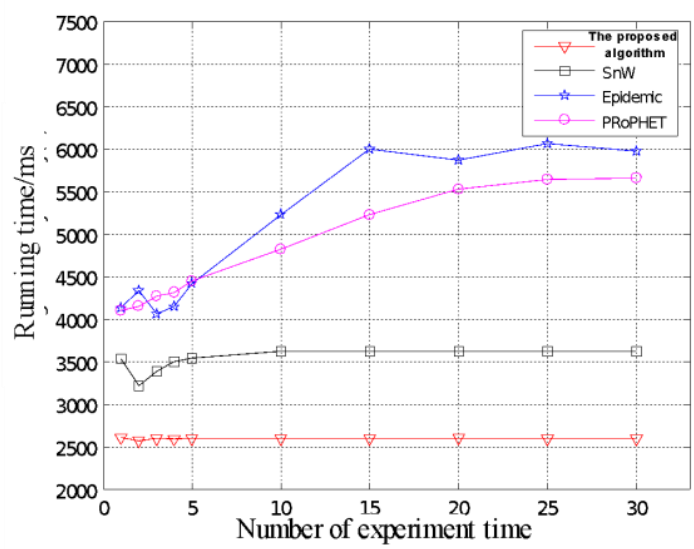

(b)

(b) Comparison of the algorithm performance for obstacle avoidance distance measurement

According to the above experimental environment and parameter description, the trajectory simulation of robot obstacle avoidance is carried out. The simulation results are shown in Figure 7. It can be inferred that the algorithm can be used to measure the obstacle avoidance distance of the robot and the trajectory of the robot to effectively avoid the obstacle, and realize the shortest and optimal distance. In order to quantitatively analyze and compare the superiority of the algorithm, based on the same obstacles and environmental settings, the time of robot running from starting point to the end point is the test parameter. Under different experiment times, the test results of using the proposed algorithm and the traditional algorithm are as follows. It can be seen that the proposed algorithm used to measure the distance of robot obstacle avoidance can save the time of the robot to reach the destination and the superiority of the algorithm is demonstrated.

In order to test the performance of the distance measurement method for obstacle avoidance in a complex environment, a set of multi-obstacle points with low complexity are used to carry out the actual test experiment. The distribution of original obstacle points is shown in Figure 7. In Figure 7, the original obstacle points are scattered chaotically in the various parts of the simulation plane andan effective path to achieve obstacle avoidance cannot be found. So, in the face of the experimental environment, if using the traditional distance measurement method for obstacle avoidance, the difficulty of the system to measure the obstacle avoidance will be very large because the increased number of the obstacle points in the system directly increase the amount of calculation.

It can be seen from the comparison of the results of the above Figure 7 that it is necessary to carry out various obstacle avoidance movements by the traditional method. Using the intelligent distance measurement method of obstacle avoidance based on the complex obstacle combined with the proposed algorithm in this paper, the obstacle avoidance distance measurement can be realized in a simple way, which greatly reduces the complexity. The results of the algorithm performance tests are shown in Table 1. 


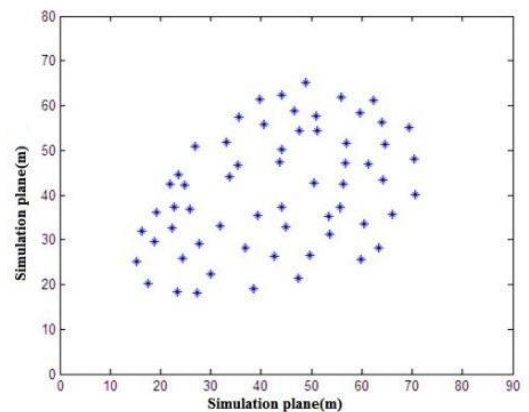

(a)

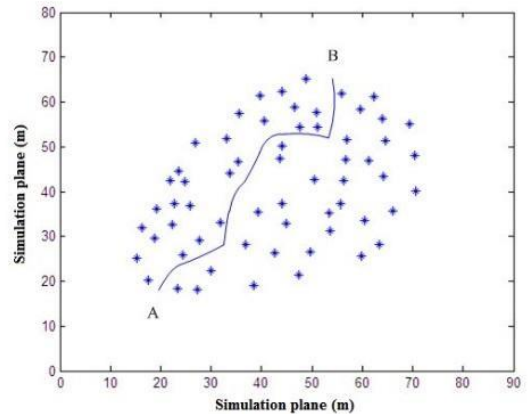

(b)

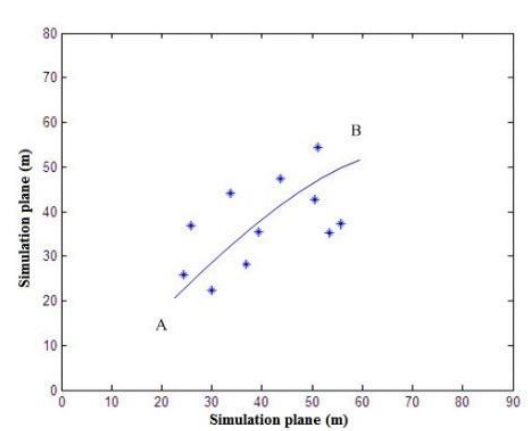

(c)

Figure 7. (a) Distribution of original obstacle points; (b) Traditional distance measurement method for obstacle avoidance; (c) Distance measurement method for obstacle avoidance in cloud computing environment

Table 1. Performance analysis of different methods for obstacle avoidance distance measurement

\begin{tabular}{|c|c|c|c|c|c|c|}
\hline \multirow{2}{*}{ Path size } & \multicolumn{2}{|c|}{ The method mentioned in this paper } & \multicolumn{2}{|c|}{ The method mentioned in reference [6] } & \multicolumn{2}{|c|}{ The method mentioned in reference [7] } \\
\hline & Number of path points & Time (seconds) & Number of path points & Time (seconds) & Number of path points & Time (seconds) \\
\hline 100 & 78 & 0.17 & 55 & 1.23 & 49 & 1.18 \\
\hline 200 & 169 & 0.22 & 132 & 2.66 & 117 & 2.12 \\
\hline 300 & 247 & 3.45 & 198 & 10.87 & 177 & 9.98 \\
\hline 400 & 349 & 4.17 & 311 & 23.54 & 303 & 22.28 \\
\hline 500 & 454 & 7.32 & 398 & 43.89 & 377 & 39.98 \\
\hline 600 & 532 & 9.98 & 478 & 61.32 & 443 & 64.78 \\
\hline 700 & 621 & 10.22 & 567 & 88.89 & 523 & 90.17 \\
\hline
\end{tabular}

It is found from the simulation results that the proposed algorithm in this paper for obstacle avoidance distance measurement is more superior to that of in reference [6] and [7] to satisfy the obstacle avoidance requirement of the robot during the actual operation. Thus, it has a broader space for development.

In order to test the performance of the proposed distance measurement algorithm of robot obstacle avoidance in the cloud computing environment, the working environment of the robot is abstracted as the grid world in Figure 8. The starting point is set as the rotating quantum gate $\mathrm{S}$, the target point is set to point $\mathrm{T}$, and obstacles are added in the environment. The optimal criterion for obstacle avoidance distance measurement in this paper is the shortest path, i.e., to find the shortest path from $\mathrm{S}$ to $\mathrm{T}$ to avoid the obstacle. A robot in any of grids in the environment can reach adjacent grids along the directions of upper, right, lower, left, upper right, lower right, lower left, and upper left.

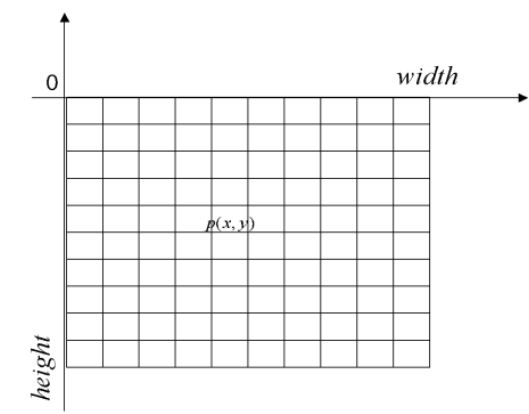

(a)

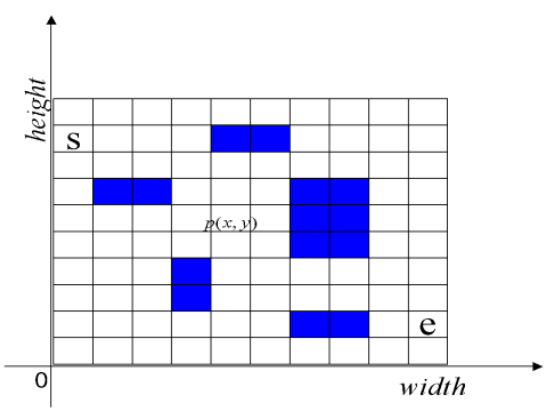

(b)

Figure 8. (a) Grid diagram of quantum evolution model; (b) Simulation environment of located robot

The simulation uses the $\mathrm{C}++$ program. The size of the map is $300 \times 300$ pixels, the unit of the length is pixel, and the working environment with irregular obstacles is simulated. The evolution process of distance measurement of robot obstacle avoidance in the complex environment is simulated and solved. The size of the model is the $300 \times 300$, the starting point is $[20,20]$, and the end point is [280, 280]. The process of finding the shortest path in the condition that there are six rectangular obstacles is shown in the following Figure 9. It can be seen that the proposed algorithm can be used to find the global optimal path.

Then, the optimal path simulation experiment of the starting point and target point is carried out using the distance measurement method of robot obstacle avoidance. When the starting point and target point of the environment are changed, the robot will quickly adapt to the new search direction according to the proposed distance measurement algorithm of 
obstacle avoidance and then obtain a new shortest path. The simulation results are shown in the following Figure 10. By changing the starting point and the end point, the distance measurement method of robot obstacle avoidance can obtain the ideal measurement results in order to achieve the robot obstacle avoidance.

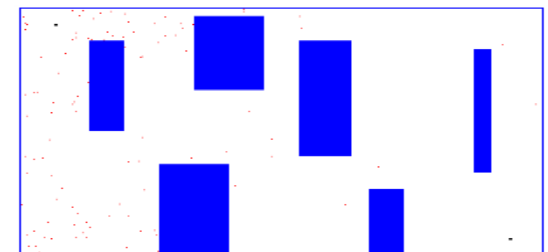

(a)

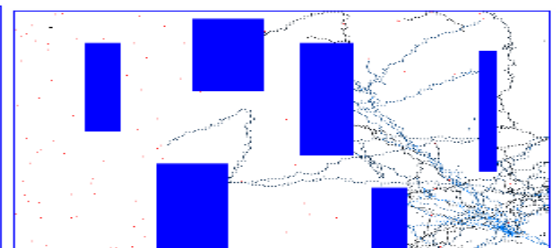

(b)

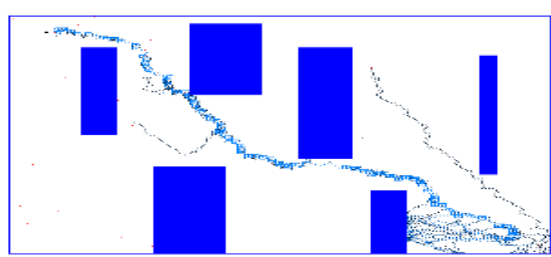

(c)

Figure 9. Location of the robot after $5 \mathrm{~s}(\mathrm{a}), 20 \mathrm{~s}$ (b) of operation, and final location (c)

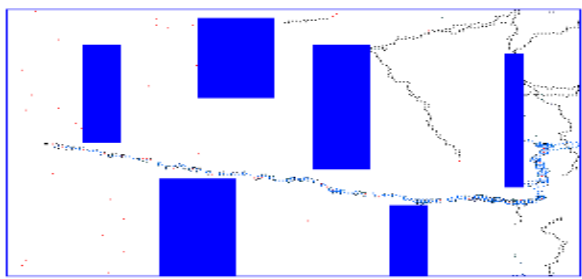

Figure 10. Path planning robot obstacle avoidance when the starting point and target point changes

The proposed algorithm in this paper is compared with that of in reference [6] and [7]. Under the same conditions, the simulation of obstacle avoidance distance measurement is carried out, and the simulation results are shown in Table 2 . It can be seen from the comparison result that the convergence speed of the proposed algorithm is improved obviously and its global optimization ability and search speed are improved remarkably, showing that this proposed algorithm has better performance in the field of robot obstacle avoidance distance measurement.

Table 2. Simulation results of different algorithms for robot obstacle avoidance distance measurement

\begin{tabular}{|c|c|c|c|c|}
\hline $\begin{array}{c}\text { Starting } \\
\text { point }\end{array}$ & $\begin{array}{c}\text { Target } \\
\text { point }\end{array}$ & $\begin{array}{c}\text { The number of iterations in this } \\
\text { paper (times) }\end{array}$ & $\begin{array}{c}\text { The number of iterations in reference } \\
{[6] \text { (times) }}\end{array}$ & $\begin{array}{c}\text { The number of iterations in reference } \\
\text { [7] (times) }\end{array}$ \\
\hline$[25,30]$ & {$[270,280]$} & 887 & 1011 & 1037 \\
\hline$[40,45]$ & {$[220,240]$} & 786 & 998 & 946 \\
\hline$[30,40]$ & {$[280,290]$} & 556 & 675 & 683 \\
\hline$[45,55]$ & {$[300,300]$} & 858 & 987 & 99 \\
\hline$[65,75]$ & {$[350,380]$} & 476 & 565 & \\
\hline
\end{tabular}

\section{Conclusions}

Aiming at the problems existing in the process of distance measurement of robot obstacle avoidance, an intelligent distance measurement method of robot obstacle avoidance in cloud computing environment is proposed. Based on DSP and ultrasonic global positioning system, a multi-channel ultrasonic transmitter/receiver module is adopted to design an autonomous obstacle avoidance control system based on ultrasonic wave and to propose a new fuzzy reasoning method to realize the function of intelligent distance measurement of robot obstacle avoidance in the cloud computing environment. The simulation and field test for intelligent distance measurement system of the obstacle avoidance are carried out by the Visual C++ visual programming software. The reliability and feasibility of the system are verified, which provides a wider space for the research and development of the robot and has a higher use value.

\section{Acknowledgements}

The research is supported by Key Scientific and Technological Support Projects of Tianjin Key R\&D Program (S18ZC58678), and Tianjin University Innovation Team Training Program (TD13-5094).

\section{References}

1. M. Zhao and B. Han, "The Research of Autonomous Obstacle Avoidance of Mobile Robot based on Multi-Sensor Integration," in Proceedings of Advanced Sensor Systems and Applications VII, pp. 1002514, 2017

2. M. Mujahed, D. Fischer, and B. Mertsching, "Tangential Gap Flow (TGF) Navigation: A new Reactive Obstacle Avoidance Approach for Highly Cluttered Environments," Robotics and Autonomous Systems, Vol. 84, pp. 15-30, 2016

3. M. S. Aman, M. A. Mahmud, H. Jiang, A. Abdelgawad, and K. Yelamarthi, "A Sensor Fusion Methodology for Obstacle 
Avoidance robot," in Proceedings of 2016 IEEE International Conference on Electro Information Technology (EIT), pp. 04580463, 2016

4. M. Sreekumar, "A Robot Manipulator with Adaptive Fuzzy Controller in Obstacle Avoidance," Journal of the Institution of Engineers (India): Series C, Vol. 97, pp. 469-478, 2016

5. S. Liu, W. Fu, W. Zhao, J. Zhou, and Q. Li, "A Novel Fusion Method by Static and Moving Facial Capture," Mathematical Problems in Engineering, Vol. 2013, pp. 503924, 2013

6. J. W. Park, H. J. Kwak, Y. C. Kang, and D. W. Kim, "Advanced Fuzzy Potential Field Method for Mobile Robot Obstacle Avoidance," Computational Intelligence and Neuroscience, Vol. 2016, pp. 10, 2016

7. M. Benzaoui, H. Chekireb, M. Tadjine, and A. Boulkroune, "Trajectory Tracking with Obstacle Avoidance of Redundant Manipulator based on Fuzzy Inference Systems," Neurocomputing, Vol. 196, pp. 23-30, 2016

8. R. C. Luo and C.-W. Kuo, "Intelligent Seven-DoF Robot with Dynamic Obstacle Avoidance and 3-D Object Recognition for Industrial Cyber-Physical Systems in Manufacturing Automation," Proceedings of the IEEE, Vol. 104, pp. 1102-1113, 2016

9. R. Ismail, Z. Omar, and S. Suaibun, "Obstacle-Avoiding Robot with IR and PIR Motion Sensors," in Proceedings of IOP Conference Series: Materials Science and Engineering, pp. 012064, 2016

10. Y. Cai and S. X. Yang, "A PSO-based Approach with Fuzzy Obstacle Avoidance for Cooperative Multi-Robots in Unknown Environments," International Journal of Computational Intelligence and Applications, Vol. 15, pp. 1650001, 2016

11. Z. Ji, H. Zhu, H. Liu, N. Liu, T. Chen, Z. Yang, et al., "The Design and Characterization of a Flexible Tactile Sensing Array for Robot Skin," Sensors, Vol. 16, pp. 2001, 2016

12. M. Wang, J. Luo, and U. Walter, "A Non-Linear Model Predictive Controller with Obstacle Avoidance for a Space Robot," Advances in Space Research, Vol. 57, pp. 1737-1746, 2016

13. C. -T. Yen and M. -F. Cheng, "A Study of Fuzzy Control with Ant Colony Algorithm Used in Mobile Robot for Shortest Path Planning and Obstacle Avoidance," Microsystem Technologies, Vol. 24, pp. 125-135, 2018

14. A. Yorozu and M. Takahashi, "Obstacle Avoidance with Translational and Efficient Rotational Motion Control Considering Movable Gaps and Footprint for Autonomous Mobile Robot," International Journal of Control, Automation and Systems, Vol. 14, pp. 1352-1364, 2016

15. H. Zhang, X. Han, M. Fu, and W. Zhou, "Robot Obstacle Avoidance Learning based on Mixture Models," Journal of Robotics, Vol. 2016, 2016

16. B. Jia, S. Liu, and Y. Yang, "Fractal Cross-Layer Service with Integration and Interaction in Internet of Things," International Journal of Distributed Sensor Networks, Vol. 10, pp. 760248, 2014

17. T. P. Nascimento, A. G. Conceiçao, and A. P. Moreira, "Multi-Robot Nonlinear Model Predictive Formation Control: The Obstacle Avoidance Problem," Robotica, Vol. 34, pp. 549-567, 2016

18. H. Gao, Q. Wei, Y. Yu, and J. Liu, "Mobile Robot Obstacle Avoidance Algorithms based on Information Fusion of Vision and Sonar," International Journal of Future Generation Communication and Networking, Vol. 9, pp. 111-120, 2016

19. S. A. Sebi and D. Sunny, "Obstacle Avoidance in Mobile Robotic Sensors and Establishing Connection," Procedia Technology, Vol. 25, pp. 364-371, 2016

20. S. Liu, W. Fu, H. Deng, C. Lan, and J. Zhou, "Distributional Fractal Creating Algorithm in Parallel Environment," International Journal of Distributed Sensor Networks, Vol. 9, pp. 281707, 2013 